\title{
Inertial Gradient-Like Dynamical System Controlled by a Stabilizing Term ${ }^{1}$
}

\author{
A. CABOT $^{2}$
}

\begin{abstract}
Let $H$ be a real Hilbert space and let $\Phi: H \rightarrow \mathbb{R}$ be a $\mathscr{C}^{1}$ function that we wish to minimize. For any potential $U: H \rightarrow \mathbb{R}$ and any control function $\epsilon: \mathbb{R}_{+} \rightarrow \mathbb{R}_{+}$which tends to zero as $t \rightarrow+\infty$, we study the asymptotic behavior of the trajectories of the following dissipative system:
\end{abstract}

$$
\text { (S) } \ddot{x}(t)+\gamma \dot{x}(t)+\nabla \Phi(x(t))+\epsilon(t) \nabla U(x(t))=0, \quad \gamma>0 .
$$

The (S) system can be viewed as a classical heavy ball with friction equation (Refs. 1-2) plus the control term $\epsilon(t) \nabla U(x(t))$. If $\Phi$ is convex and $\epsilon(t)$ tends to zero fast enough, each trajectory of (S) converges weakly to some element of $\operatorname{argmin} \Phi$. This is a generalization of the Alvarez theorem (Ref. 1). On the other hand, assuming that $\epsilon$ is a slow control and that $\Phi$ and $U$ are convex, the (S) trajectories tend to minimize $U$ over $\operatorname{argmin} \Phi$ when $t \rightarrow+\infty$. This asymptotic selection property generalizes a result due to Attouch and Czarnecki (Ref. 3) in the case where $U(x)=$ $|x|^{2} / 2$. A large part of our results are stated for the following wider class of systems:

$$
\text { (GS) } \ddot{x}(t)+\gamma \dot{x}(t)+\nabla_{x} \Psi(t, x(t))=0,
$$

where $\Psi: \mathbb{R}_{+} \times H \rightarrow \mathbb{R}$ is a $C^{1}$ function.

Key Words. Dissipative dynamical systems, nonlinear oscillators, optimization, convex minimization, heavy ball with friction.

\footnotetext{
${ }^{1}$ The author expresses his gratitude to Marc-Olivier Czarnecki for help during the realization of this work. The author thanks the referees for their careful reading of the paper. Their valuable suggestions, critical remarks, and pertinent comments led to numerous improvements throughout.

${ }^{2}$ Assistant Professor, Laboratory LACO, Faculty of Science, University of Limoges, Limoges, France.
} 


\section{Introduction}

Let $H$ be a real Hilbert space, with scalar product and corresponding norm respectively denoted by $\langle\cdot, \cdot\rangle$ and $|\cdot|$. Let $\Phi: H \rightarrow \mathbb{R}$ be a given $\mathscr{C}^{1}$ realvalued function, called the potential function. In order to reach the minima of $\Phi$, a powerful method consists in following the trajectories of a dissipative dynamical gradient-like system as $t \rightarrow \infty$.

Let $\Sigma \subset H \times \mathbb{R}$ be the manifold defined by $\Sigma:=\operatorname{Graph}(\Phi)$ and let us consider the motion of a material point with unit mass, subjected to stay on $\Sigma$ and which moves under the action of the gravity force, the reaction force, and the friction force $(\gamma>0$ is the friction parameter). A simple application of the fundamental principle of dynamics and a reasonable approximation (Ref. 4) lead to the so-called "heavy ball with friction" system (Refs. 1-2)

$(\mathrm{HBF}) \quad \ddot{x}(t)+\gamma \dot{x}(t)+\nabla \Phi(x(t))=0, \quad t \geq 0$.

The (HBF) system is dissipative and can be studied in the classical framework of the theory of dissipative dynamical systems [see for example, Hale (Ref. 5) and Haraux (Ref. 6)]. It enjoys most of the nice properties of the steepest descent method. To quote only one of them, when $\Phi$ is convex, the Brezis-Bruck Theorem (Refs. 7-8) has been extended by Alvarez to (HBF). The main interest of (HBF) lies in the fact that it has more exploration properties than a first-order in time dynamical system.

Coming back to the mechanical interpretation, assume that the material point is subjected to extra forces deriving from a time-dependent potential of the form $\epsilon(t) U$, where $U: H \rightarrow \mathbb{R}$ is a $\mathscr{C}^{1}$ function and $\epsilon: \mathbb{R}_{+} \rightarrow \mathbb{R}_{+}$tends to zero as $t \rightarrow+\infty$. In such a situation, (HBF) turns into

(S) $\ddot{x}(t)+\gamma \dot{x}(t)+\nabla \Phi(x(t))+\epsilon(t) \nabla U(x(t))=0$.

In this paper, we are concerned mainly with the asymptotic behavior of the (S) trajectories. The function $\epsilon$ acts as a control on the trajectories of (S). When $\epsilon$ tends to zero fast enough, more precisely if

$$
\int_{0}^{+\infty} \epsilon(t) d t<+\infty,
$$

the study of (S) is quite similar to that of (HBF), which corresponds to the noncontrolled case $(\epsilon \equiv 0)$. In particular, one can extend the Alvarez theorem (Ref. 1) with arguments relying on the Opial lemma (Ref. 9). The situation is much more involved when dealing with a slow control, namely,

$$
\int_{0}^{+\infty} \epsilon(t) d t=+\infty .
$$


Some variants of the (S) system have been studied by several authors. For example in Ref. 3, Attouch and Czarnecki couple the Tikhonov regularization with the (HBF) dynamical system. They prove that, when $\Phi$ is convex and $\epsilon: \mathbb{R}_{+} \rightarrow \mathbb{R}_{+}$is a $\mathscr{C}^{1}$ control function such that $\int_{0}^{+\infty} \epsilon(t) d t=+\infty$, then each trajectory of the system

$$
\left((\mathrm{S}) \text { with } U(x)=|x|^{2} / 2\right) \quad \ddot{x}(t)+\gamma \dot{x}(t)+\nabla \Phi(x(t))+\epsilon(t) x(t)=0
$$

strongly converges to the point of minimal norm of $\operatorname{argmin} \Phi$. The condition

$$
\int_{0}^{+\infty} \epsilon(t) d t=+\infty
$$

expresses that $\epsilon(t)$ tends to zero slowly enough to allow the Tikhonov regularization term $\epsilon(t) x(t)$ to be effective asymptotically.

In another direction, Cabot and Czarnecki consider in Ref. 10 a particular case of the (S) system where

$$
H=\mathscr{H}^{2}, \quad \Phi(x, y)=\phi(x)+\phi(y), \quad U(x, y)=V(x-y),
$$

thus leading to

$$
\begin{aligned}
& \ddot{x}(t)+\gamma \dot{x}(t)+\nabla \phi(x(t))+\epsilon(t) \nabla V(x-y)(t)=0, \\
& \ddot{y}(t)+\gamma \dot{y}(t)+\nabla \phi(y(t))-\epsilon(t) \nabla V(x-y)(t)=0 .
\end{aligned}
$$

They focus their attention on the case where the potential $V$ modelizes a repulsion force. Such a potential allows a better exploration of the minima of $\phi$ than a (simple) (HBF) method. In Ref. 10, Cabot and Czarnecki show that the condition

$$
\int_{0}^{+\infty} \epsilon(t) d t=+\infty
$$

plays once more an essential role in order to make the repulsion efficient.

Coming back to the (S) system and assuming that $\epsilon$ is a slow control [i.e., $\int_{0}^{+\infty} \epsilon(t) d t=+\infty$ ], we prove in this paper a general asymptotic selection result: under convex conditions on $\Phi$ and $U$, the trajectories of (S) tend to minimize the function $U$ over the set $\operatorname{argmin} \Phi$ when $t \rightarrow+\infty$. This last property shows the potential interest of the (S) system in convex constrained optimization.

The study of (S) can be placed in a more general setting by introducing the function

$$
\Psi(t, x)=\Phi(x)+\epsilon(t) U(x)
$$


then, the system (S) can be rewritten as

(GS) $\ddot{x}(t)+\gamma \dot{x}(t)+\nabla_{x} \Psi(t, x(t))=0$,

where $G$ stands for "generalized". This system is very interesting from a theoretical point of view in the sense that it recovers a large number of situations. A first-order in time version of (GS) has been studied by Attouch and Cominetti (Ref. 11), who have coupled approximation methods with the steepest descent system. When dealing with (GS), one can define for each fixed $t$ the default of convexity $\omega(t)$ of the map $\Psi(t, \cdot)$. This quantity can play partially the part of the term $\epsilon(t)$ of (S) in the study of (GS). In this paper, we will state our first convergence results in the frame of (GS) and then, when necessary, we will particularize to (S).

The paper is organized as follows. In Section 2, we develop the mechanical interpretation of the (S) system. In Section 3, we establish general existence properties for (S) and (GS). In Section 4, we give our first convergence results in the case of a fast control (i.e., tending to zero sufficiently fastly). Section 5 is devoted to the asymptotic behavior of the (S) trajectories when the control $\epsilon$ is slow, giving rise to the minimization of $U$ over the set $\operatorname{argmin} \Phi$. The proofs of the main results of the paper are postponed to Section 6.

\section{2. (S) and (GS) Systems in Mechanics and Optimization}

2.1. Mechanical Origin. Let $\mathscr{H}$ be a real Hilbert space with scalar product and corresponding norm respectively denoted by $\langle\cdot, \cdot\rangle_{\mathscr{H}}$ and $|\cdot|_{\mathscr{H}}$. Let $\phi: \mathscr{H} \rightarrow \mathbb{R}$ be a given function of class $\mathscr{C}^{1}$ that has to be minimized. The heavy ball with friction method (Ref. 2) consists in studying the following system:

(HBF) $\quad \ddot{x}(t)+\gamma \dot{x}(t)+\nabla \phi(x(t))=0, \quad t \geq 0$,

where $\gamma>0$ is a positive damping parameter. Let us consider now $N \geq 2$ heavy balls coupled by an interaction force. We assume that the $i$ th oscillator exerts on the $j$ th one a force $f_{i j}$ equal to

$$
f_{i j}=-\epsilon(t) \nabla V\left(x_{j}-x_{i}\right),
$$

where $V: \mathscr{H} \rightarrow \mathbb{R}$ is a $\mathscr{C}^{1}$ function such that

$$
\nabla V(-x)=-\nabla V(x), \quad \text { for all } x \in \mathscr{H},
$$


so that $f_{i j}=-f_{j i}$, and $\epsilon: \mathbb{R}_{+} \rightarrow \mathbb{R}$ is a time-dependent function. Then, the trajectories $x_{i}(\cdot)_{1 \leq i \leq N}$ satisfy the relations

$$
\begin{aligned}
& \ddot{x}_{1}(t)+\gamma \dot{x}_{1}(t)+\nabla \phi\left(x_{1}(t)\right)+\epsilon(t) \sum_{j \neq 1} \nabla V\left(x_{1}(t)-x_{j}(t)\right)=0, \\
& \ddot{x}_{i}(t)+\gamma \dot{x}_{i}(t)+\nabla \phi\left(x_{i}(t)\right)+\epsilon(t) \sum_{j \neq i} \nabla V\left(x_{i}(t)-x_{j}(t)\right) \quad=0, \\
& \ddot{x}_{N}(t)+\gamma \dot{x}_{N}(t)+\nabla \phi\left(x_{N}(t)\right)+\epsilon(t) \sum_{j \neq N} \nabla V\left(x_{N}(t)-x_{j}(t)\right)=0 .
\end{aligned}
$$

Setting

$$
\begin{aligned}
& H=\mathscr{H}^{N}, \\
& x=\left(x_{1}, \ldots, x_{N}\right) \in H, \\
& \Phi(x)=\sum_{i=1}^{N} \phi\left(x_{i}\right), \\
& U(x)=\sum_{\substack{i, j=1 \\
i<j}}^{N} V\left(x_{i}-x_{j}\right),
\end{aligned}
$$

we can rewrite easily the previous system as a unique equation on $H$,

$$
\ddot{x}(t)+\gamma \dot{x}(t)+\nabla \Phi(x(t))+\epsilon(t) \nabla U(x(t))=0 .
$$

2.2. (S) and (GS) Systems. Let $H$ be a real Hilbert space with scalar product and corresponding norm respectively denoted by $\langle\cdot, \cdot\rangle$ and $|\cdot|$. Let $\Phi: H \rightarrow \mathbb{R}$ and $U: H \rightarrow \mathbb{R}$ be functions of class $\mathscr{C}^{1}$ and let $\epsilon: \mathbb{R}_{+} \rightarrow \mathbb{R}_{+}$be a map which tends to 0 when $t \rightarrow+\infty$. In the whole paper, we are interested in the following dynamical system:

(S) $\quad \ddot{x}(t)+\gamma \dot{x}(t)+\nabla \Phi(x(t))+\epsilon(t) \nabla U(x(t))=0, \quad t \geq 0$.

A first example of the (S) system comes from the mechanical model developed in Section 2.1. This corresponds to the situation where the global interaction is the sum of the interaction between pairs. Such a system with a repulsion force has been studied by Cabot and Czarnecki (Ref. 10) in the case $N=2$. The interest in optimization of such a repulsion force comes from the fact that it allows a better exploration of the local minima.

Another important situation corresponds to the case where $U(x)=$ $|x|^{2} / 2$. Then, the (S) system reduces to

$$
\ddot{x}(t)+\gamma \dot{x}(t)+\nabla \Phi(x(t))+\epsilon(t) x(t)=0 .
$$


As a matter of fact, the term $\epsilon(t) x(t)$ has a stabilizing effect and is linked closely to the Tikhonov regularization (Ref. 12). This system has been studied intensively by Attouch and Czarnecki (Ref. 3).

The study of (S) can be placed in a more general setting by introducing the function

$$
\Psi(t, x)=\Phi(x)+\epsilon(t) U(x) .
$$

Then, the system (S) can be rewritten as

(GS) $\ddot{x}(t)+\gamma \dot{x}(t)+\nabla_{x} \Psi(t, x(t))=0$,

where $G$ stands for "generalized". In the following section, we investigate the general properties of (S) and (GS) by using classical techniques in the study of differential equations.

\section{General Properties of (S) and (GS)}

Let $H$ be a real Hilbert space. Let us consider a mapping $\Psi: \mathbb{R}_{+} \times H \rightarrow \mathbb{R}$ of class $\mathscr{C}^{1}$ which satisfies the following conditions:

(A1) $\Psi(\cdot, x)$ is a nonincreasing function; i.e., $\partial \Psi / \partial t \leq 0$.

(A2) $\Psi$ is bounded from below on $\mathbb{R}_{+} \times H$.

Moreover, assume the following Lipschitz-like conditions on $\nabla_{x} \Psi$ :

(A3) For every bounded set $B$ of $H$, there exists $K_{B} \geq 0$ such that $\left|\nabla_{x} \Psi(t, y)-\nabla_{x} \Psi(t, x)\right| \leq K_{B}|y-x|, \quad \forall t \in \mathbb{R}_{+}, \quad \forall x, y \in B$.

(A4) For every bounded set $B$ of $H$, there exists a function $L_{B}: \mathbb{R}_{+} \rightarrow \mathbb{R}$ such that $\sup _{\mathbb{R}_{+}} L_{B}<+\infty$ and

$$
\begin{aligned}
& \left|\nabla_{x} \Psi(t+h, x)-\nabla_{x} \Psi(t, x)\right| \leq L_{B}(t+h)-L_{B}(t), \\
& \forall x \in B, \quad \forall t, h \in \mathbb{R}_{+} .
\end{aligned}
$$

Then, let us consider the following dynamical system:

(GS) $\ddot{x}(t)+\gamma \dot{x}(t)+\nabla_{x} \Psi(t, x(t))=0$.

The mechanical energy of (GS), defined by

$$
E(t)=(1 / 2)|\dot{x}(t)|^{2}+\Psi(t, x(t)),
$$

provides us with a natural Lyapunov function. Indeed, a simple computation gives

$$
\begin{aligned}
\dot{E}(t) & =\langle\dot{x}(t), \ddot{x}(t)\rangle+(\partial / \partial t) \Psi(t, x(t))+\left\langle\dot{x}(t), \nabla_{x} \Psi(t, x(t))\right\rangle . \\
& =-\gamma|\dot{x}(t)|^{2}+(\partial / \partial t) \Psi(t, x(t)) .
\end{aligned}
$$


From Assumption (A1), we have

$$
(\partial / \partial t) \Psi(t, x(t)) \leq 0
$$

hence,

$$
\dot{E}(t) \leq 0 ;
$$

i.e., $E$ is nonincreasing. The existence of a Lyapunov function for a differential equation is useful in the study of the asymptotic stability of its equilibria. Lyapunov methods and other power tools (like the Lasalle invariance principle) have been developed to study such a question. We refer to the abundant literature on this subject (Ref. 6 and Refs. 13-16). The central result of this section is given by the following theorem, whose proof relies mainly on the existence of the Lyapunov function $E$.

Theorem 3.1. Let us assume that $\Psi: \mathbb{R}_{+} \times H \rightarrow \mathbb{R}$ satisfies Assumptions (A1) to (A4) and that the parameter $\gamma$ is positive. Then, the following properties hold:

(i) For all $\left(x_{0}, \dot{x}_{0}\right)$ in $H \times H$, there exists a unique maximal solution $x: \mathbb{R}_{+} \rightarrow H$ of (GS) which is of class $\mathscr{C}^{2}$ on $[0,+\infty[$ and satisfies the initial conditions $x(0)=x_{0}$ and $\dot{x}(0)=\dot{x}_{0}$.

(ii) For every trajectory $x(\cdot)$ of (GS), the following estimation holds: $\dot{x} \in L^{\infty}([0,+\infty) ; H) \cap L^{2}([0,+\infty) ; H)$.

(iii) Moreover, assuming that $x$ is bounded [i.e., $\left.x \in L^{\infty}([0,+\infty) ; H)\right]$, then we have

$$
\begin{aligned}
& \lim _{t \rightarrow+\infty} \dot{x}(t)=0 \quad \text { and } \quad \lim _{t \rightarrow+\infty} \ddot{x}(t)=0, \\
& \lim _{t \rightarrow+\infty} \nabla_{x} \Psi(t, x(t))=0 .
\end{aligned}
$$

Proof. The proof of (i) is a classical application of the CauchyLipschitz theorem. The $L^{\infty}$ and $L^{2}$ estimates of $\dot{x}$ are immediate consequences of the decay of the Lyapunov function $E$. The details of these proofs are left to the reader. The proof of (iii) is postponed to Section 6.1.

Corollary 3.1. Under the assumptions of Theorem 3.1, assume moreover that the function $\Phi(\cdot)=\inf _{t \geq 0} \Psi(t, \cdot)$ is coercive, i.e., $\lim _{|x| \rightarrow+\infty} \Phi(x)=$ $+\infty$. Then, $x \in L^{\infty}([0,+\infty) ; H)$ and the conclusions of Theorem 3.1 hold.

\section{Proof. Using}

$$
\Phi(x(t)) \leq \Psi(t, x(t))
$$


and the decay of the function $E$, we obtain

$$
(1 / 2)|\dot{x}(t)|^{2}+\Phi(x(t)) \leq E(t) \leq E(0)
$$

and we deduce that

$$
\Phi(x(t)) \leq E(0) .
$$

This majorization on $\Phi(x(t))$ and the coerciveness of $\Phi$ imply that $x \in L^{\infty}([0,+\infty) ; H)$.

Applications to the (S) System. As an application of the previous results, we consider now the case where $\Psi$ is of the form

$$
\Psi(t, x)=\Phi(x)-\inf \Phi+\epsilon(t)(U(x)-\inf U) .
$$

The functions $\Phi: H \rightarrow \mathbb{R}, U: H \rightarrow \mathbb{R}$, and $\epsilon: \mathbb{R}_{+} \rightarrow \mathbb{R}_{+}$are of class $\mathscr{C}^{1}$, and satisfy respectively:

(B1) $\Phi$ is bounded from below on $H$.

(B2) $\nabla \Phi$ is Lipschitz continuous on the bounded subsets of $H$.

(C1) $U$ is bounded from below on $H$.

(C2) $\nabla U$ is Lipschitz continuous on bounded subsets of $H$.

(D1) $\lim _{t \rightarrow+\infty} \epsilon(t)=0$.

(D2) The function $\epsilon$ is nonincreasing; i.e., $\dot{\epsilon}(t) \leq 0, \forall t \in \mathbb{R}_{+}$.

Then, a simple application of Theorem 3.1 gives the following proposition.

Proposition 3.1. Let us consider the differential system

(S) $\ddot{x}(t)+\gamma \dot{x}(t)+\nabla \Phi(x)(t)+\epsilon(t) \nabla U(x(t))=0$,

where $\Phi, U, \epsilon$ satisfy respectively Assumptions (B), (C), (D). Then, we have the same conclusions (i), (ii), (iii) as in Theorem 3.1. Moreover, if $x \in L^{\infty}([0,+\infty) ; H)$, then $\lim _{t \rightarrow+\infty} \nabla \Phi(x(t))=0$.

\section{Proof. Setting}

$$
\Psi(t, x)=\Phi(x)-\inf \Phi+\epsilon(t)(U(x)-\inf U),
$$

it is sufficient to prove that Assumptions (A1) to (A4) of Theorem 3.1 are satisfied. This is immediate and is left to the reader.

When $\Phi$ is strongly convex, the convergence of the gradient toward 0 yields immediately the strong convergence of the trajectory. 
Proposition 3.2. In addition to the assumptions of Proposition 3.1, let us assume that $\Phi$ is strongly convex; that is, for any $R>0$, there exists a function $\beta_{R}: \mathbb{R}_{+} \rightarrow \mathbb{R}_{+}$, with $\beta_{R}\left(t_{n}\right) \rightarrow 0 \Rightarrow t_{n} \rightarrow 0$, such that

$\langle\nabla \Phi(x)-\nabla \Phi(y), x-y\rangle \geq \beta_{R}(|x-y|), \quad \forall x, y \in H$ with $|x|<R,|y|<R$.

If the trajectory $x(\cdot)$ of $(\mathrm{S})$ is bounded [i.e., $\left.x \in L^{\infty}([0,+\infty) ; H)\right]$, then it is norm convergent as $t$ goes to $+\infty$ to the unique global minimizer $\bar{x}$ of $\Phi$.

Proof. The proof is an immediate consequence of the fact that

$$
\lim _{t \rightarrow+\infty} \nabla \Phi(x(t))=0,
$$

combined with the strong convexity property (1) of $\Phi$.

\section{Convergence of (S) and (GS) Trajectories: Case of a Fast Control}

In the previous section, we have shown the global existence of (GS) trajectories and the convergence of the gradients toward zero. When the potential $\Phi$ is convex, Alvarez has proved in Ref. 1 that the trajectories of the system

$$
\text { (HBF) } \ddot{x}(t)+\gamma \dot{x}(t)+\nabla \Phi(x)(t)=0
$$

weakly converge to a minimum of $\Phi$. To extend this result to (GS), we appeal to the notion of convexity default. More precisely, we assume that:

(A5) For every bounded set $B$ of $H$, there exists $\omega_{B}: \mathbb{R}_{+} \rightarrow \mathbb{R}_{+}$such that for every $t \geq 0$,

$$
\left\langle\nabla_{x} \Psi(t, y)-\nabla_{x} \Psi(t, x), y-x\right\rangle \geq-\omega_{B}(t)|y-x|^{2}, \quad \forall x, y \in B .
$$

The quantity $\omega_{B}(t)$ is called the convexity default of $\Psi(t, \cdot)$ on $B$ and can be characterized equivalently by the following inequality: for every $\lambda \in[0,1]$ and every $x, y \in B$,

$$
\begin{aligned}
& (1-\lambda) \Psi(t, x)+\lambda \Psi(t, y) \\
& \geq \Psi(t,(1-\lambda) x+\lambda y)-\left[\omega_{B}(t) / 2\right] \lambda(1-\lambda)|y-x|^{2},
\end{aligned}
$$

which in turn is equivalent to

$$
\Psi(t, y) \geq \Psi(t, x)+\left\langle\nabla_{x} \Psi(t, x), y-x\right\rangle-\left[\omega_{B}(t) / 2\right]|y-x|^{2} .
$$

These characterizations of the convexity default will be useful in the sequel. The proof of their equivalence is classical and is left to the reader. 
We assume that the convexity default tends to zero fast enough, more precisely:

(A6) For every bounded set $B$ of $H$,

$$
\lim _{t \rightarrow+\infty} \omega_{B}(t)=0 \text { and } \int_{0}^{+\infty} \omega_{B}(t) d t<+\infty .
$$

Remark 4.1. If the map $\Psi(t, \cdot)$ is convex for each fixed $t$, one can take $\omega_{B} \equiv 0$ and then Assumptions (A5) and (A6) are satisfied automatically.

Assumptions (A5) and (A6) imply that the function $\Phi(\cdot)=\lim _{t \rightarrow+\infty}$ $\Psi(t, \cdot)$ is convex continuous on $H$, as stated by the following proposition.

Proposition 4.1. Under Assumptions (A1) to (A6), the function $\Phi(\cdot)=\lim _{t \rightarrow+\infty} \Psi(t, \cdot)$ is convex continuous on $H$.

Proof. Let $B$ be a bounded convex set of $H$. Using the characterization (3) of the convexity default of $\Psi(t, \cdot)$ on $B$ and passing to the limit when $t \rightarrow+\infty$ by taking into account the fact that

$$
\lim _{t \rightarrow+\infty} \omega_{B}(t)=0,
$$

then we obtain

$$
(1-\lambda) \Phi(x)+\lambda \Phi(y) \geq \Phi((1-\lambda) x+\lambda y), \quad \forall \lambda \in[0,1], \quad \forall x, y \in B ;
$$

i.e., $\Phi$ is convex on $B$. This being true for any bounded convex set $B$, the map $\Phi$ is convex on $H$.

Let us now prove that $\Phi$ is continuous on $H$. From Assumption (A1), the map $\Phi$ is the lower envelope of the functions $(\Psi(t, \cdot))_{t \geq 0}$. Therefore, $\Phi$ is upper semicontinuous on $H$, hence locally bounded from above on $H$. From a classical result, the convex function $\Phi$ is then continuous on $H$.

We assume lastly that:

(A7) $\operatorname{argmin} \Phi \neq \emptyset$ and, for every $z \in \operatorname{argmin} \Phi, \int_{0}^{+\infty}\left|\nabla_{x} \Psi(t, z)\right| d t<+\infty$.

We can state now the following theorem.

Theorem 4.1. Let us assume that $\Psi: \mathbb{R}_{+} \times H \rightarrow \mathbb{R}$ satisfies Assumptions (A1) to (A7). Let $\Phi$ denote the convex function defined by $\Phi(\cdot)=\lim _{t \rightarrow+\infty}$ $\Psi(t, \cdot)$ and let $x(\cdot)$ be the unique solution of (GS). If $x \in L^{\infty}([0,+\infty) ; H)$, then there exists $x_{\infty} \in \operatorname{argmin} \Phi$ such that

$$
w-\lim _{t \rightarrow+\infty} x(t)=x_{\infty} .
$$


The proof of Theorem 4.1 uses mainly the Opial lemma and is given in Section 6.2.

Applications to the (S) System. We apply now the previous result to the case where

$$
\Psi(t, x)=\Phi(x)-\inf \Phi+\epsilon(t)(U(x)-\inf U) .
$$

In this framework, Assumptions (A5)-(A6) are equivalent to the fact that $\Phi$ is convex and that $\epsilon$ tends to 0 fast enough,

$$
\int_{0}^{+\infty} \epsilon(t) d t<+\infty, \quad \text { fast control. }
$$

Then, one can deduce from Theorem 4.1 the following proposition.

Proposition 4.2. In addition to Hypotheses (B), (C), (D), let us assume that

(E1) The map $\Phi$ is convex and $\operatorname{argmin} \Phi \neq \emptyset$.

(E2) $\int_{0}^{+\infty} \epsilon(t) d t<+\infty$ (fast control).

Let $x(\cdot)$ be the unique solution of (S). If $x \in L^{\infty}([0,+\infty) ; H)$, then there exists $x_{\infty} \in \operatorname{argmin} \Phi$ such that

$$
w-\lim _{t \rightarrow+\infty} x(t)=x_{\infty} .
$$

\section{Proof. Setting}

$$
\Psi(t, x)=\Phi(x)-\inf \Phi+\epsilon(t)[U(x)-\inf U],
$$

we have just to verify that Assumptions (A5) to (A7) of Theorem 4.1 are satisfied. This simple verification is left to the reader.

Let us remark that, in the previous result, the boundedness assumption on $x(\cdot)$ takes a fundamental place. If $\Phi$ is coercive, this assumption is verified automatically. We are going now to examine a sufficient condition on $U$ which ensures that the map $x$ is bounded. Let us state the following proposition.

Proposition 4.3. Under the assumptions of Proposition 4.2, let $x$ be the unique solution of the (S) system. Moreover, we assume that $\nabla U$ has at most a linear growth, i.e.,

$$
|\nabla U(x)| \leq A+B|x|, \quad \forall x \in H,
$$


for some positive $A$ and $B$. Then, $x \in L^{\infty}([0,+\infty) ; H)$; hence, the conclusions of Proposition 4.2 apply.

The proof of Proposition 4.3 uses Gronwall type arguments and is given in Section 6.3.

\section{Case of a Slow Control: Minimization of $U$ over $\operatorname{argmin} \Phi$}

5.1. Case of a Convex Potential. Attouch and Czarnecki (Ref. 3) have considered the particular case of (S) where

$$
U(x)=|x|^{2} / 2,
$$

thus leading to

$$
\ddot{x}(t)+\gamma \dot{x}(t)+\nabla \Phi(x(t))+\epsilon(t) x(t)=0 .
$$

When $\Phi$ is convex and $\epsilon: \mathbb{R}_{+} \rightarrow \mathbb{R}_{+}$tends to zero slowly, i.e.,

$$
\int_{0}^{+\infty} \epsilon(t) d t=+\infty
$$

they have proved that each trajectory of (5) converges strongly to the point of minimal norm of the set $S=\operatorname{argmin} \Phi$.

Our purpose in the following theorem is to generalize the result of Attouch and Czarnecki (Ref. 3) to the case where $U$ is a convex function such that $\operatorname{argmin}_{S} U$ is reduced to a singleton $\{p\}, p \in H$. For example, this situation occurs when $U$ is strictly convex. Then, the following theorem shows the convergence of the (S) trajectories toward the point $p$.

Theorem 5.1. In addition to Hypotheses (B), (C), (D), let us assume that $H=\mathbb{R}^{n}$ and that:

(F1) $\Phi: H \rightarrow \mathbb{R}$ and $U: H \rightarrow \mathbb{R}$ are convex and $S:=\operatorname{argmin} \Phi \neq \emptyset$.

(F2) The set $\operatorname{argmin}_{S} U$ is reduced to a singleton $\{p\}, p \in H$.

(F3) $\int_{0}^{+\infty} \epsilon(t) d t=+\infty$ (slow control).

If the trajectory $x(\cdot)$ of the (S) system is bounded [i.e., $\left.x \in L^{\infty}([0,+\infty) ; H)\right]$, then it converges to $p$, namely, $\lim _{t \rightarrow+\infty} x(t)=p$.

Theorem 5.1 is proved in Section 6.4. The condition

$$
\int_{0}^{+\infty} \epsilon(t) d t=+\infty
$$

corresponds to the fact that $\epsilon(t)$ does not tend to zero too rapidly, thus allowing the regularization term $\epsilon(t) \nabla U(x(t))$ to be effective asymptotically. This result shows an asymptotic selection property: the effect of such a slow 
control $\epsilon$ forces all the trajectories to converge to the same equilibrium $p$. This situation contrasts sharply with the noncontrolled situation or fast control, where the limits of the trajectories depend on the initial data, and may also be difficult to identify.

Beside the regularization role of the term $\epsilon(t) \nabla U(x)$, the (S) system can be useful in the framework of convex constrained minimization. Indeed, given a convex set $S \subset H$ and a convex function $U: H \rightarrow \mathbb{R}$, consider the following general minimization problem:

(P) $\min \{U(x), x \in S\}$.

We define now a convex function $\Phi: H \rightarrow \mathbb{R}$ satisfying $\operatorname{argmin} \Phi=S$ [for instance, $\left.\Phi=(1 / 2) d_{S}^{2}\right]$ and a control parameter $\epsilon: \mathbb{R}_{+} \rightarrow \mathbb{R}_{+}$such that

$$
\int_{0}^{+\infty} \epsilon(t) d t=+\infty
$$

When $(\mathrm{P})$ has a unique solution $p$, Theorem 5.1 tells us that the trajectories of (S) converge to $p$. Figure 1 illustrates this situation with $S=\mathbb{R}_{+}^{2}$ and

$$
U(x, y)=(1 / 2)\left[4(x+y+1)^{2}+(x-y-1)^{2}\right] .
$$

The $(\mathrm{S})$ trajectory is computed with the following data:

$$
\begin{array}{lll}
\Phi(x, y)=(1 / 2) d_{S}^{2}(x, y), & \epsilon(t)=1 /(1+t), & \left(x_{0}, y_{0}\right)=(5,3), \\
\left(\dot{x}_{0}, \dot{y}_{0}\right)=(0,0), & \gamma=1 . &
\end{array}
$$

When $(\mathrm{P})$ has a set of nonisolated solutions, the behavior at infinity of the (S) trajectories is still an open problem. From this point of view, Theorem 5.1 can be viewed as a first step when considering the (S) system as a tool for convex constrained minimization.

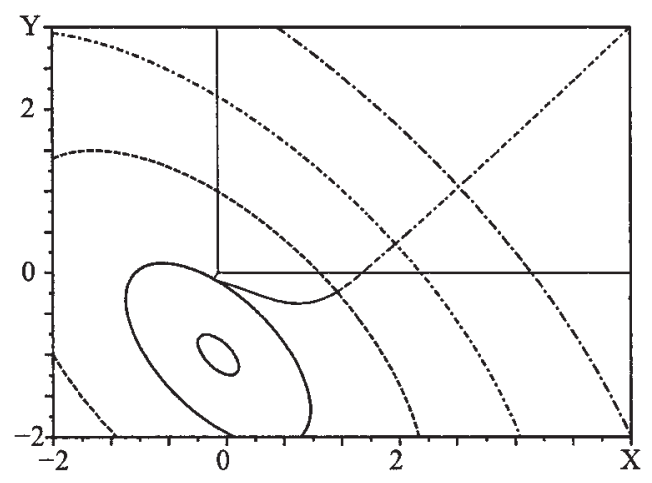

Fig. 1. Convergence of the (S) trajectory toward $\operatorname{argmin}_{S} U$. 
We give now a sufficient condition which ensures that the (S) trajectory is bounded.

Proposition 5.1. Under the assumptions of Theorem 5.1, let $x(\cdot)$ be the unique solution of the (S) system. Assume that, for every $M \in \mathbb{R}$, the following condition holds:

(G) The set $\left\{x \in H, U(x) \leq \min _{S} U\right\} \cap\{x \in H, \Phi(x) \leq M\}$ is bounded.

Then, $x \in L^{\infty}([0,+\infty) ; H)$; hence, in view of Theorem 5.1,

$$
\lim _{t \rightarrow+\infty} x(t)=p \text {. }
$$

Moreover, condition $(\mathrm{G})$ is satisfied in each of the following cases:

(a) $\Phi$ is coercive; i.e., $\lim _{|x| \rightarrow+\infty} \Phi(x)=+\infty$.

(b) $U$ is coercive; i.e., $\lim _{|x| \rightarrow+\infty} U(x)=+\infty$.

The proof of Proposition 5.1 is postponed to Section 6.5.

5.2. Toward a Nonconvex Potential. Let us consider now the mechanical model developed in Section 2.1. In the particular case $N=2$, it leads to the following system on $H=\mathscr{H} \times \mathscr{H}$ :

$$
\begin{aligned}
& \ddot{x}(t)+\gamma \dot{x}(t)+\nabla \phi(x(t))+\epsilon(t) \nabla V(x(t)-y(t))=0, \\
& \ddot{y}(t)+\gamma \dot{y}(t)+\nabla \phi(y(t))-\epsilon(t) \nabla V(x(t)-y(t))=0 .
\end{aligned}
$$

Moreover, assume that $V$ modelizes a repulsion potential; i.e.,

$$
\langle\nabla V(z), z\rangle_{\mathscr{H}} \leq 0, \quad \forall z \in \mathscr{H} .
$$

It is easy to verify that, in general, such a function $V$ and the associated function

$$
U(x, y)=V(x-y)
$$

are not convex. As a consequence, Theorem 5.1 does not apply and one has to perform a specific study. In this direction, Cabot and Czarnecki (Ref. 10) have proved an interesting one-dimensional result. More precisely, when $\mathscr{H}=\mathbb{R}$, assume that $\phi: \mathbb{R} \rightarrow \mathbb{R}$ is convex, that $V: \mathbb{R} \rightarrow \mathbb{R}$ satisfies (7), and that $\epsilon$ is a slow control. Setting

$$
\operatorname{argmin} \phi=[a, b], \quad-\infty \leq a \leq b \leq+\infty,
$$

Cabot and Czarnecki have proved that the solution $(x, y)$ of $(6)$ satisfies one of the following cases:

(i) $\lim _{t \rightarrow+\infty}(x(t), y(t))=(a, b)$;

(ii) $\lim _{t \rightarrow+\infty}(x(t), y(t))=(b, a)$;

(iii) $\lim _{t \rightarrow+\infty} x(t)=\lim _{t \rightarrow+\infty} y(t) \in[a, b]$. 
Case (i) [resp. (ii)] just expresses that $\left(\lim _{t \rightarrow+\infty} x(t), \lim _{t \rightarrow+\infty} y(t)\right) \in \mathbb{R}^{2}$ is a local minimum point on $S=[a, b]^{2}$ for the function

$$
(x, y) \mapsto U(x, y)=V(x-y) .
$$

Case (iii) is the pathological one and, after numerical experiments, Cabot and Czarnecki have conjectured that the set of initial conditions leading to this case is negligible. As a consequence, we may conjecture the following generalization of Theorem 5.1: when $\epsilon$ is a slow control, for almost every initial condition $\left(x_{0}, \dot{x}_{0}\right) \in H^{2}$, the trajectory $x(\cdot)$ of (S) tends to minimize at least locally the (possibly) nonconvex function $U$ on the set $S$.

\section{Proof of the Main Results}

6.1. Proof of Theorem 3.1(iii). We assume that $x \in L^{\infty}([0,+\infty) ; H)$; i.e., there exists a bounded set $B$ such that $x(\cdot) \subset B$. Let us first notice that it is sufficient to prove that

$$
\lim _{t \rightarrow+\infty} \ddot{x}(t)=0 .
$$

Indeed, in that case, the function $h:=\dot{x}$ satisfies both the relations

$$
h \in L^{2}([0,+\infty) ; H) \text { and } \quad \dot{h} \in L^{\infty}([0,+\infty) ; H) .
$$

According to a classical result, these two properties imply

$$
\lim _{t \rightarrow+\infty} h(t)=0
$$

i.e.,

$$
\lim _{t \rightarrow+\infty} \dot{x}(t)=0 .
$$

Then, in view of (GS), we infer immediately that

$$
\lim _{t \rightarrow+\infty} \nabla_{x} \Psi(t, x(t))=0 .
$$

Let us now prove that

$$
\lim _{t \rightarrow+\infty} \ddot{x}(t)=0 .
$$

For any $h>0$, let us define

$$
u_{h}(t)=(1 / h)[\dot{x}(t+h)-\dot{x}(t)] .
$$

The proof consists in showing that $u_{h}(t)$ converges to 0 , uniformly with respect to $h$. Let us write the (GS) equation at the points $t$ and $t+h$; let us 
make the difference and divide by $h$. We obtain

$$
\dot{u}_{h}(t)+\gamma u_{h}(t)=f_{h}(t)
$$

where

$$
f_{h}(t)=-\left[\nabla_{x} \Psi(t+h, x(t+h))-\nabla_{x} \Psi(t, x(t))\right] / h .
$$

The function $f_{h}$ can be decomposed as

$$
f_{h}=f_{1 h}+f_{2 h},
$$

where

$$
\begin{aligned}
& f_{1 h}(t)=-\left[\nabla_{x} \Psi(t+h, x(t+h))-\nabla_{x} \Psi(t+h, x(t))\right] / h, \\
& f_{2 h}(t)=-\left[\nabla_{x} \Psi(t+h, x(t))-\nabla_{x} \Psi(t, x(t))\right] / h .
\end{aligned}
$$

Let us now multiply (8) by $e^{\gamma s}$ and integrate between $t^{\prime}$ and $t$, obtaining

$$
u_{h}(t)=u_{h}\left(t^{\prime}\right) e^{-\gamma\left(t-t^{\prime}\right)}+e^{-\gamma t} \int_{t^{\prime}}^{t} e^{\gamma s} f_{h}(s) d s,
$$

implying that

$\left|u_{h}(t)\right| \leq\left|u_{h}\left(t^{\prime}\right)\right| e^{-\gamma\left(t-t^{\prime}\right)}+e^{-\gamma t} \int_{t^{\prime}}^{t} e^{\gamma s}\left|f_{1 h}(s)\right| d s+e^{-\gamma t} \int_{t^{\prime}}^{t} e^{\gamma s}\left|f_{2 h}(s)\right| d s$.

In view of (A3), we have

$$
\begin{aligned}
\left|f_{1 h}(s)\right| & \leq K_{B}[|x(s+h)-x(s)| / h] \\
& \leq\left(K_{B} / h\right) \int_{s}^{s+h}|\dot{x}(u)| d u,
\end{aligned}
$$

and from the Cauchy-Schwarz inequality we obtain

$$
\left|f_{1 h}(s)\right|^{2} \leq\left(K_{B}^{2} / h\right) \int_{s}^{s+h}|\dot{x}(u)|^{2} d u .
$$

Using again the Cauchy-Schwarz inequality, we can find now an upper bound for $e^{-\gamma t} \int_{t^{t}}^{t} e^{\gamma_{s}}\left|f_{1 h}(s)\right| d s$, specifically,

$$
\begin{aligned}
e^{-\gamma t} \int_{t^{\prime}}^{t} e^{\gamma s}\left|f_{1 h}(s)\right| d s & \leq e^{-\gamma t}\left(\int_{t^{\prime}}^{t} e^{2 \gamma s} d s\right)^{1 / 2}\left(\int_{t^{\prime}}^{t}\left|f_{1 h}(s)\right|^{2} d s\right)^{1 / 2} \\
& \leq\left(K_{B} / \sqrt{2 \gamma}\right)\left(\int_{t^{\prime}}^{+\infty}(1 / h) \int_{s}^{s+h}|\dot{x}(u)|^{2} d u d s\right)^{1 / 2} .
\end{aligned}
$$

On the other hand, Assumption (A4) implies

$$
\left|f_{2 h}(s)\right| \leq\left[L_{B}(s+h)-L_{B}(s)\right] / h,
$$


and hence,

$$
e^{-\gamma t} \int_{t^{\prime}}^{t} e^{\gamma s}\left|f_{2 h}(s)\right| d s \leq \int_{t^{\prime}}^{+\infty}\left\{\left[L_{B}(s+h)-L_{B}(s)\right] / h\right\} d s .
$$

We need now the following lemma.

Lemma 6.1. Let $g: \mathbb{R}_{+} \rightarrow \mathbb{R}$ be a nondecreasing function satisfying $\sup _{\mathbb{R}_{+}} g<+\infty$. Then, for every $h>0$ and $t \geq 0$,

$$
\int_{t}^{+\infty}\{[g(s+h)-g(s)] / h\} d s \leq \lim _{s \rightarrow+\infty} g(s)-g(t) .
$$

Proof. Let $T \geq t$. Then,

$$
\begin{aligned}
\int_{t}^{T}\{[g(s+h)-g(s)] / h\} d s & =(1 / h) \int_{t+h}^{T+h} g(s) d s-(1 / h) \int_{t}^{T} g(s) d s \\
& =(1 / h) \int_{T}^{T+h} g(s) d s-(1 / h) \int_{t}^{t+h} g(s) d s .
\end{aligned}
$$

Since $g$ is nondecreasing, the last equality implies

$$
\int_{t}^{T}\{[g(s+h)-g(s)] / h\} d s \leq g(T+h)-g(t) .
$$

Taking the limit when $T \rightarrow+\infty$, we obtain the result.

Combining (9)-(11) and applying Lemma 6.1 to the functions $s \mapsto L_{B}(s)$ and $s \mapsto \int_{0}^{s}|\dot{x}(u)|^{2} d u$, we obtain finally $\left|u_{h}(t)\right| \leq\left|u_{h}\left(t^{\prime}\right)\right| e^{-\gamma\left(t-t^{\prime}\right)}+\left(K_{B} / \sqrt{2 \gamma}\right)\left(\int_{t^{\prime}}^{+\infty}|\dot{x}(s)|^{2} d s\right)^{1 / 2}+\lim _{s \rightarrow+\infty} L_{B}(s)-L_{B}\left(t^{\prime}\right)$.

Notice that $u_{h}\left(t^{\prime}\right)$ is uniformly bounded with respect to $h$. Indeed, from part (ii), the map $\dot{x}$ is bounded and

$$
\lim _{h \rightarrow 0} u_{h}\left(t^{\prime}\right)=\ddot{x}\left(t^{\prime}\right) .
$$

Then, taking the supremum in $h$ and the upper limit when $t \rightarrow+\infty$, we deduce from the last inequality that

$$
\limsup _{t \rightarrow+\infty}\left[\sup _{h>0}\left|u_{h}(t)\right|\right] \leq\left(K_{B} / \sqrt{2 \gamma}\right)\left(\int_{t^{\prime}}^{+\infty}|\dot{x}(s)|^{2} d s\right)^{1 / 2}+\lim _{s \rightarrow+\infty} L_{B}(s)-L_{B}\left(t^{\prime}\right) .
$$


Taking the limit when $t^{\prime} \rightarrow+\infty$, we obtain

$$
\lim _{t \rightarrow+\infty}\left[\sup _{h>0}\left|u_{h}(t)\right|\right]=0 .
$$

Since, for all $t \geq 0$,

$$
|\ddot{x}(t)| \leq \sup _{h>0}\left|u_{h}(t)\right|,
$$

we conclude that

$$
\lim _{t \rightarrow+\infty} \ddot{x}(t)=0 \text {. }
$$

6.2. Proof of Theorem 4.1. The central idea is to prove the weak convergence of the trajectory $x$ by using the Opial lemma (Ref. 9).

Lemma 6.2. See Ref. 9. Let $H$ be a Hilbert space and let $x:[0,+\infty[\rightarrow H$ be a function such that there exists a nonvoid set $S \subset H$ which verifies:

(i) $\forall z \in S, \lim _{t \rightarrow+\infty}|x(t)-z|$ exists.

(ii) $\forall t_{n} \rightarrow+\infty$ with $x\left(t_{n}\right) \rightarrow x_{\infty}$ weakly in $H$, we have $x_{\infty} \in S$.

Then, $x(t)$ weakly converges as $t \rightarrow+\infty$ to some element $x_{\infty}$ of $S$.

Let us apply the Opial lemma with $S=\operatorname{argmin} \Phi$.

(i) For any $z \in \operatorname{argmin} \Phi \neq \emptyset$, let us set

$$
h(t)=\langle\dot{x}(t), x(t)-z\rangle+(\gamma / 2)|x(t)-z|^{2} .
$$

Then, we have

$$
\begin{aligned}
\dot{h}(t) & =|\dot{x}(t)|^{2}+\langle x(t)-z, \ddot{x}(t)\rangle+\gamma\langle x(t)-z, \dot{x}(t)\rangle \\
& =|\dot{x}(t)|^{2}-\left\langle x(t)-z, \nabla_{x} \Psi(t, x(t))\right\rangle \\
& =|\dot{x}(t)|^{2}-\left\langle x(t)-z, \nabla_{x} \Psi(t, x(t))-\nabla_{x} \Psi(t, z)\right\rangle-\left\langle x(t)-z, \nabla_{x} \Psi(t, z)\right\rangle .
\end{aligned}
$$

By Assumption (A5), for every $t \geq 0$,

$$
\left\langle x(t)-z, \nabla_{x} \Psi(t, x(t))-\nabla_{x} \Psi(t, z)\right\rangle \geq-\omega_{B}(t)|x(t)-z|^{2},
$$

where $B$ is a bounded set of $H$ containing $z$ and the trajectory $x(\cdot)$ $\left[x \in L^{\infty}([0,+\infty) ; H)\right.$, by assumption]. Hence,

$$
\begin{aligned}
\dot{h}(t) & \leq|\dot{x}(t)|^{2}+\omega_{B}(t)|x(t)-z|^{2}+|x(t)-z|\left|\nabla_{x} \Psi(t, z)\right| \\
& \leq|\dot{x}(t)|^{2}+C^{2} \omega_{B}(t)+C\left|\nabla_{x} \Psi(t, z)\right|,
\end{aligned}
$$

where $C$ is an upper bound for $|x(t)-z|$. 
From Theorem 3.1, we have

$$
|\dot{x}|^{2} \in L^{1}(0,+\infty) \text {. }
$$

On the other hand, because of (A6) and (A7),

$$
\omega_{B}(\cdot) \in L^{1}(0,+\infty) \text { and }\left|\nabla_{x} \Psi(., z)\right| \in L^{1}(0,+\infty) .
$$

Taking the positive part of the previous relation, we obtain finally

$$
\dot{h}_{+}(t) \leq|\dot{x}(t)|^{2}+C^{2} \omega_{B}(t)+C\left|\nabla_{x} \Psi(t, z)\right| \in L^{1}(0,+\infty) .
$$

Then, we can apply the following lemma.

Lemma 6.3. Let $h: \mathbb{R}_{+} \rightarrow \mathbb{R}$ be a function of class $\mathscr{C}^{1}$ which is bounded from below and such that $\dot{h}_{+} \in L^{1}(0,+\infty)$. Then, $\lim _{t \rightarrow+\infty} h(t)$ exists. Hence,

$$
\lim _{t \rightarrow+\infty} h(t)=\lim _{t \rightarrow+\infty}\langle\dot{x}(t), x(t)-z\rangle+(\gamma / 2)|x(t)-z|^{2} \quad \text { exists. }
$$

Since

$$
x \in L^{\infty}([0,+\infty) ; H) \text { and } \lim _{t \rightarrow+\infty} \dot{x}(t)=0,
$$

we conclude that

$$
\lim _{t \rightarrow+\infty}|x(t)-z| \quad \text { exists. }
$$

(ii) Let us now consider a sequence $\left(t_{n}\right)$ such that

$$
w-\lim _{n \rightarrow+\infty} x\left(t_{n}\right)=x_{\infty} .
$$

We have to prove that

$$
x_{\infty} \in \operatorname{argmin} \Phi .
$$

Let us fix $y \in H$ and consider a bounded set $B$ containing $y$ and the trajectory $x(\cdot)$. Using the characterization (4) of the convexity default of $\Psi(t, \cdot)$ on $B$, we deduce that

$$
\begin{aligned}
& \Psi\left(t_{n}, y\right)-\Psi\left(t_{n}, x\left(t_{n}\right)\right) \\
& \geq\left\langle\nabla_{x} \Psi\left(t_{n}, x\left(t_{n}\right)\right), y-x\left(t_{n}\right)\right\rangle-\left[\omega_{B}\left(t_{n}\right) / 2\right]\left|y-x\left(t_{n}\right)\right|^{2} .
\end{aligned}
$$

Passing to the upper limit in (12), we obtain

$$
\Phi(y)-\liminf _{n \rightarrow+\infty} \Psi\left(t_{n}, x\left(t_{n}\right)\right) \geq 0,
$$

because

$$
\lim _{t \rightarrow+\infty} \nabla_{x} \Psi(t, x(t))=0 \quad \text { (cf. Theorem 3.1) }
$$


and

$$
\lim _{t \rightarrow+\infty} \omega_{B}(t)=0 \quad[\mathrm{cf} .(\mathrm{A} 6)]
$$

Using (13), we find that

$$
\Phi(y) \geq \liminf _{n \rightarrow+\infty} \Psi\left(t_{n}, x\left(t_{n}\right)\right) \geq \liminf _{n \rightarrow+\infty} \Phi\left(x\left(t_{n}\right)\right) \geq \Phi\left(x_{\infty}\right) .
$$

The last inequality comes from the fact that $\Phi$ is convex continuous (cf. Proposition 4.1), hence 1.s.c. for the weak topology on $H$. This being true for any $y \in H$, we have proved that

$$
x_{\infty} \in \operatorname{argmin} \Phi \text {. }
$$

The Opial lemma allows us to conclude that there exists $x_{\infty} \in \operatorname{argmin} \Phi$ such that

$$
w-\lim _{t \rightarrow+\infty} x(t)=x_{\infty}
$$

6.3. Proof of Proposition 4.3. For any $z \in \operatorname{argmin} \Phi$, let us set

$$
h(t):=\langle\dot{x}(t), x(t)-z\rangle+(\gamma / 2)|x(t)-z|^{2} .
$$

Since $x$ is solution of $(\mathrm{S})$ and $\Phi$ is convex, we have

$$
\begin{aligned}
\dot{h}(t) & =|\dot{x}(t)|^{2}+\langle x(t)-z, \ddot{x}(t)+\gamma \dot{x}(t)\rangle \\
& =|\dot{x}(t)|^{2}-\langle x(t)-z, \nabla \Phi(x(t))\rangle-\epsilon(t)\langle x(t)-z, \nabla U(x(t))\rangle \\
& \leq|\dot{x}(t)|^{2}-\epsilon(t)\langle x(t)-z, \nabla U(x(t))\rangle \\
& \leq|\dot{x}(t)|^{2}+\epsilon(t)|x(t)-z||\nabla U(x(t))| .
\end{aligned}
$$

Since $\nabla U$ has at most a linear growth, we deduce that

$$
\begin{aligned}
\dot{h}(t) & \leq|\dot{x}(t)|^{2}+A \epsilon(t)|x(t)-z|+B \epsilon(t)|x(t)-z||x(t)| \\
& \leq|\dot{x}(t)|^{2}+(A+B|z|) \epsilon(t)|x(t)-z|+B \epsilon(t)|x(t)-z|^{2} .
\end{aligned}
$$

Let us use the classical inequality

$$
\alpha \leq \alpha^{2}+1 / 4
$$

to obtain

$$
\begin{aligned}
\dot{h}(t) & \leq|\dot{x}(t)|^{2}+[(A+B|z|) / 4] \epsilon(t)+(A+B|z|+B) \epsilon(t)|x(t)-z|^{2} \\
& \leq|\dot{x}(t)|^{2}+C \epsilon(t)+C^{\prime} \epsilon(t)|x(t)-z|^{2} .
\end{aligned}
$$


We integrate the previous inequality on $[0, t]$, obtaining

$$
\begin{aligned}
h(t) & \leq h(0)+\int_{0}^{t}|\dot{x}(s)|^{2} d s+C \int_{0}^{t} \epsilon(s) d s+C^{\prime} \int_{0}^{t} \epsilon(s)|x(s)-z|^{2} d s \\
& \leq h(0)+\int_{0}^{+\infty}|\dot{x}(s)|^{2} d s+C \int_{0}^{+\infty} \epsilon(s) d s+C^{\prime} \int_{0}^{t} \epsilon(s)|x(s)-z|^{2} d s .
\end{aligned}
$$

On the other hand, we have

$$
\begin{aligned}
h(t) & =(\gamma / 4)|x(t)-z|^{2}+(\gamma / 4)\left[|x(t)-z|^{2}+2\langle(2 / \gamma) \dot{x}(t), x(t)-z\rangle\right] \\
& \geq(\gamma / 4)|x(t)-z|^{2}-(\gamma / 4)\left[\left(4 / \gamma^{2}\right)|\dot{x}(t)|^{2}\right] \\
& \geq(\gamma / 4)|x(t)-z|^{2}-\|\dot{x}\|_{\infty}^{2} / \gamma .
\end{aligned}
$$

Combining (14) and (15), we obtain

$$
\begin{aligned}
& (\gamma / 4)|x(t)-z|^{2} \\
& \leq\|\dot{x}\|_{\infty}^{2} / \gamma+h(0)+\int_{0}^{+\infty}|\dot{x}(s)|^{2} d s+C \int_{0}^{+\infty} \epsilon(s) d s+C^{\prime} \int_{0}^{t} \epsilon(s)|x(s)-z|^{2} d s .
\end{aligned}
$$

Setting

$$
C^{\prime \prime}=\|\dot{x}\|_{\infty}^{2} / \gamma+h(0)+\int_{0}^{+\infty}|\dot{x}(s)|^{2} d s+C \int_{0}^{+\infty} \epsilon(s) d s,
$$

we can apply now the Gronwall lemma to the function $|x-z|^{2}$ and we find that

$$
\begin{aligned}
|x(t)-z|^{2} & \leq\left(4 C^{\prime \prime} / \gamma\right) \exp \left[\left(4 C^{\prime} / \gamma\right) \int_{0}^{t} \epsilon(s) d s\right] \\
& \leq\left(4 C^{\prime \prime} / \gamma\right) \exp \left[\left(4 C^{\prime} / \gamma\right) \int_{0}^{+\infty} \epsilon(s) d s\right] .
\end{aligned}
$$

The right side is finite and hence

$$
x \in L^{\infty}([0,+\infty) ; H) .
$$

6.4. Proof of Theorem 5.1. The proof is an extension of the arguments of Attouch and Czarnecki (Ref. 3). In our situation, it relies on the study of the function $h$ defined by

$$
h(t)=\langle\dot{x}(t), x(t)-p\rangle+(\gamma / 2)|x(t)-p|^{2},
$$

where

$$
p:=\operatorname{argmin}_{S} U .
$$

We have to prove that $h$, converges to 0 . By differentiating $h$, we find that

$$
\dot{h}(t)=|\dot{x}(t)|^{2}-\langle x(t)-p, \nabla \Phi(x(t))\rangle-\epsilon(t)\langle x(t)-p, \nabla U(x(t))\rangle .
$$


From the convexity of $\Phi$,

$$
p \in \operatorname{argmin} \Phi \text { implies }\langle p-x(t), \nabla \Phi(x(t))\rangle \leq 0 ;
$$

hence,

$$
\dot{h}(t) \leq|\dot{x}(t)|^{2}+\epsilon(t)\langle p-x(t), \nabla U(x(t))\rangle .
$$

The main idea of the proof is now to distinguish respectively the cases where

$$
\langle p-x(t), \nabla U(x(t))\rangle 0\rangle \quad \text { and }\langle p-x(t), \nabla U(x(t))\rangle \leq 0 .
$$

Precisely, we distinguish three cases:

(a) $\exists T \geq 0, \forall t \geq T,\langle p-x(t), \nabla U(x(t))\rangle \leq 0$;

(b) $\exists T \geq 0, \forall t \geq T,\langle p-x(t), \nabla U(x(t))\rangle>0$;

(c) $\forall T \geq 0, \exists t \geq T,\langle p-x(t), \nabla U(x(t))\rangle>0$.

Case (c) obviously contains Case (b), but the main points of the proof are made clearer with this distinction.

In the whole proof, we use the following notation:

$$
E_{p}:=\{x \in H,\langle p-x, \nabla U(x)\rangle \geq 0\} .
$$

Let us state a first claim which will be useful in the sequel.

Claim 6.1. Under the assumptions of Theorem 5.1, we have $E_{p} \cap S=\{p\}$.

Proof. Let $x \in E_{p} \cap S$; from the definition of $E_{p}$, we have

$$
\langle p-x, \nabla U(x)\rangle \geq 0,
$$

which combined with the convexity of $U$ yields

$$
U(p) \geq U(x) \quad \text { i.e., } \min _{S} U \geq U(x) .
$$

Since $x \in S$, we conclude that $x \in \operatorname{argmin}_{S} U=\{p\}$.

Case (a). We assume that there is $T \geq 0$ such that, for every $t \geq T$,

$$
\langle p-x(t), \nabla U(x(t))\rangle \leq 0 ;
$$

hence, from (16), we deduce that, for every $t \geq T$,

$$
\dot{h}(t) \leq|\dot{x}(t)|^{2} .
$$


Taking the positive part of the previous inequality, we obtain $\dot{h}_{+} \in L^{1}(0,+\infty)$ and, using Lemma 6.3, this implies that

$$
\lim _{t \rightarrow+\infty} h(t) \quad \text { exists. }
$$

We now prove that

$$
\lim _{t \rightarrow+\infty} h(t)=0 .
$$

Assume that it is not true. Then, $\lim _{t \rightarrow+\infty} h(t)$ exists and is strictly positive. Let us set

$$
\alpha:=\lim _{t \rightarrow+\infty}|x(t)-p|>0 .
$$

Let us first prove that

$$
\limsup _{t \rightarrow+\infty}\langle p-x(t), \nabla U(x(t))\rangle<0 .
$$

Indeed, if it is not true, there exists a sequence $\left(t_{n}\right)$ such that

$$
\lim _{n \rightarrow+\infty} t_{n}=+\infty \text { and } \lim _{n \rightarrow+\infty}\left\langle p-x\left(t_{n}\right), \nabla U\left(x\left(t_{n}\right)\right)\right\rangle=0 .
$$

Since the function $x$ is bounded, without loss of generality we may assume that there is $\bar{x} \in H$ such that $x\left(t_{n}\right)$ converges to $\bar{x}$. At the limit, when $n \rightarrow+\infty$, we obtain that

$$
\langle p-\bar{x}, \nabla U(\bar{x})\rangle=0,
$$

which implies $\bar{x} \in E_{p}$. From Proposition 3.1, we get that

$$
\lim _{n \rightarrow+\infty} \nabla \Phi\left(x\left(t_{n}\right)\right)=0,
$$

and hence $\bar{x} \in S$. Finally,

$$
\bar{x} \in E_{p} \cap S=\{p\} .
$$

On the other hand, we have $|\bar{x}-p|=\alpha>0$, a contradiction. As a conclusion,

$$
\limsup _{t \rightarrow+\infty}\langle p-x(t), \nabla U(x(t))\rangle<0 ;
$$

i.e., there exists $l>0$ and $t_{1}>0$ such that, for every $t \geq t_{1}$,

$$
\langle p-x(t), \nabla U(x(t))\rangle \leq-l .
$$

Hence,

$$
\dot{h}(t) \leq|\dot{x}(t)|^{2}-\epsilon(t) l .
$$


Thus, by integrating between $t_{1}$ and $t$ and passing to the limit when $t \rightarrow+\infty$, we get

$$
\lim _{t \rightarrow+\infty} h(t)+l \int_{t_{1}}^{+\infty} \epsilon(s) d s \leq h\left(t_{1}\right)+\int_{t_{1}}^{+\infty}|\dot{x}(s)|^{2} d s,
$$

which contradicts the fact that

$$
|\dot{x}|^{2} \in L^{1}(0,+\infty) \quad \text { and } \quad \epsilon \notin L^{1}(0,+\infty) .
$$

Hence,

$$
\lim _{t \rightarrow+\infty} h(t)=\lim _{t \rightarrow+\infty}\langle\dot{x}(t), x(t)-p\rangle+(\gamma / 2)|x(t)-p|^{2}=0 .
$$

Since

$$
\lim _{t \rightarrow+\infty} \dot{x}(t)=0,
$$

we conclude that

$$
\lim _{t \rightarrow+\infty}|x(t)-p|=0 .
$$

Case (b). We assume that there is $T \geq 0$ such that, for every $t \geq T$,

$$
\langle p-x(t), \nabla U(x(t))\rangle>0
$$

and hence, for every $t \geq T$,

$$
x(t) \in E_{p} .
$$

Since $x(\cdot)$ is bounded, from Proposition 3.1, we get that

$$
\lim _{t \rightarrow+\infty} \nabla \Phi(x(t))=0 .
$$

Considering a subsequence $x\left(t_{n}\right)$, then we have

$$
\lim _{n \rightarrow+\infty} \nabla \Phi\left(x\left(t_{n}\right)\right)=0 .
$$

Applying the following claim to the sequence $\left(x\left(t_{n}\right)\right)$, we obtain that

$$
\lim _{n \rightarrow+\infty} x\left(t_{n}\right)=p,
$$

which concludes the proof of Case (b).

Claim 6.2. Let $\left(x_{n}\right)$ be a bounded sequence in $E_{p}$ such that $\lim _{n \rightarrow+\infty}$ $\nabla \Phi\left(x_{n}\right)=0$. Then, $\lim _{n \rightarrow+\infty} x_{n}=p$.

Proof. Since $\left(x_{n}\right)$ is bounded, there exist $\bar{x} \in E_{p}$ and a subsequence of $\left(x_{\sigma(n)}\right)$, still denoted by $\left(x_{\sigma(n)}\right)$, such that

$$
\lim _{n \rightarrow+\infty} x_{\sigma(n)}=\bar{x} \text {. }
$$


In view of

$$
\lim _{n \rightarrow+\infty} \nabla \Phi\left(x_{\sigma(n)}\right)=0,
$$

we get that $\bar{x} \in S$. On the other hand, from Claim 6.1,

$$
E_{p} \cap S=\{p\},
$$

so that we obtain $\bar{x}=p$. Since $p$ is the limit of every convergent subsequence of $\left(x_{n}\right)_{n \geq 0}$, we deduce that the sequence $\left(x_{n}\right)_{n \geq 0}$ converges to $p$.

Case (c). We assume now that, for every $T \geq 0$, there exists some $t \geq T$ such that

$$
\langle p-x(t), \nabla U(x(t))\rangle>0 .
$$

Take any sequence $\left(t_{n}\right) \subset \mathbb{R}_{+}$such that

$$
\lim _{n \rightarrow+\infty} t_{n}=+\infty,
$$

and let us prove that

$$
\lim _{n \rightarrow+\infty} x\left(t_{n}\right)=p .
$$

First, assume that there is a subsequence $\left(t_{n}^{\prime}\right)$ of $\left(t_{n}\right)$ such that $x\left(t_{n}^{\prime}\right) \in E_{p}$. Since the map $x$ is bounded, from Proposition 3.1, we have

$$
\lim _{t \rightarrow+\infty} \nabla \Phi(x(t))=0,
$$

and hence,

$$
\lim _{n \rightarrow+\infty} \nabla \Phi\left(x\left(t_{n}^{\prime}\right)\right)=0 .
$$

Since $x\left(t_{n}^{\prime}\right) \in E_{p}$, from Claim 6.2, we deduce that

$$
\lim _{n \rightarrow+\infty} x\left(t_{n}^{\prime}\right)=p .
$$

We assume now that there is a subsequence $\left(t_{n}^{\prime \prime}\right)$ of $\left(t_{n}\right)$ such that $x\left(t_{n}^{\prime \prime}\right) \notin E_{p}$ and we prove that

$$
\lim _{n \rightarrow+\infty} x\left(t_{n}^{\prime \prime}\right)=p .
$$

We need the following claim.

Claim 6.3. Let $t \geq 0$ such that $x(t) \notin E_{p}$ and let

$$
\tau(t)=\inf \left\{u \in[0, t] \mid x([u, t]) \cap E_{p}=\emptyset\right\} .
$$


Then,

$$
h(t) \leq h(\tau(t))+\int_{\tau(t)}^{+\infty}|\dot{x}(u)|^{2} d u
$$

Proof. For every $u \in] \tau(t), t], x(u) \notin E_{p}$, that is,

$$
\langle p-x(u), \nabla U(x(u))\rangle \leq 0 .
$$

From (16), we deduce that

$$
\dot{h}(u) \leq|\dot{x}(u)|^{2} .
$$

By integrating between $\tau(t)$ and $t$, we obtain

$$
h(t)-h(\tau(t)) \leq \int_{\tau(t)}^{t}|\dot{x}(u)|^{2} d u,
$$

which yields immediately the expected formula.

We now come back to the proof of Case (c). Since $x\left(t_{n}^{\prime \prime}\right) \notin E_{p}$, let $\tau\left(t_{n}^{\prime \prime}\right)$ be defined by Claim 6.3. We notice first that

$$
\lim _{n \rightarrow+\infty} \tau\left(t_{n}^{\prime \prime}\right)=+\infty
$$

and that

$$
x\left(\tau\left(t_{n}^{\prime \prime}\right)\right) \in E_{p},
$$

for $n$ large enough. Let $n$ be large enough. Since $x\left(\tau\left(t_{n}^{\prime \prime}\right)\right) \in E_{p}$, from Claim 6.2, we have

$$
\lim _{n \rightarrow+\infty} x\left(\tau\left(t_{n}^{\prime \prime}\right)\right)=p
$$

i.e.,

$$
\lim _{n \rightarrow+\infty} h\left(\tau\left(t_{n}^{\prime \prime}\right)\right)=0 .
$$

Since $\dot{x} \in L^{2}([0,+\infty), H)$, we have also

$$
\lim _{n \rightarrow+\infty} \int_{\tau\left(t_{n}^{\prime \prime}\right)}^{+\infty}|\dot{x}(u)|^{2} d u=0 .
$$

Hence, in view of Claim 6.3, we deduce that

$$
\limsup _{n \rightarrow+\infty} h\left(t_{n}^{\prime \prime}\right) \leq 0 .
$$

On the other hand, it is clear that, because of the definition of $h$ and the fact that

$$
\lim _{n \rightarrow+\infty} \dot{x}\left(t_{n}^{\prime \prime}\right)=0,
$$


we have

$$
\limsup _{n \rightarrow+\infty} h\left(t_{n}^{\prime \prime}\right)=(\gamma / 2) \limsup _{n \rightarrow+\infty}\left|x\left(t_{n}^{\prime \prime}\right)-p\right|^{2} .
$$

Hence, we deduce that

$$
\lim _{n \rightarrow+\infty}\left|x\left(t_{n}^{\prime \prime}\right)-p\right|=0,
$$

which concludes the proof of Case (c).

6.5. Proof of Proposition 5.1. We keep here the notations of the proof of Theorem 5.1. Setting

$$
p:=\underset{S}{\operatorname{argmin}} U,
$$

we still use the function $h$ defined by

$$
h(t)=\langle\dot{x}(t), x(t)-p\rangle+(\gamma / 2)|x(t)-p|^{2}
$$

and the set

$$
E_{p}=\{x \in H,\langle p-x, \nabla U(x)\rangle \geq 0\} .
$$

From the decay of the function

$$
E(t)=(1 / 2)|\dot{x}(t)|^{2}+(\Phi(x(t))-\min \Phi)+\epsilon(t)(U(x(t))-\min U),
$$

there exists $M \in \mathbb{R}$ such that, for every $t \geq 0$,

$$
\Phi(x(t)) \leq M
$$

i.e.,

$$
\{x(t), t \geq 0\} \subset[\Phi \leq M] .
$$

On the other hand, from the convexity of $U$, we have that, for every $x \in H$,

$$
\langle p-x, \nabla U(x)\rangle \geq 0 \Rightarrow U(x) \leq U(p)=\min _{S} U
$$

therefore,

$$
E_{p} \subset\left[U \leq \min _{S} U\right] .
$$

We distinguish now the same cases (a), (b), (c) as in the proof of Theorem 5.1.

Case (a). From (17), $\lim _{t \rightarrow+\infty}|x(t)-p|$ exists, which implies that $x \in L^{\infty}([0,+\infty) ; H)$.

Case (b). The trajectory $\{x(t), t \geq T\}$ is contained in $E_{p}$; hence, from (18) and (19), it is contained in the set $\left[U \leq \min _{S} U\right] \cap[\Phi \leq M]$, which is 
bounded in view of condition $(\mathrm{G})$; as a consequence, the map $x$ is bounded; i.e., $x \in L^{\infty}([0,+\infty) ; H)$.

Case (c). Let $T \geq 0$ such that $x(T) \in E_{p}$ and consider $t \geq T$. If $x(t) \in E_{p}$, then $|x(t)-p| \leq \rho$, where $\rho$ is the diameter of the bounded set $\left[U \leq \min _{S} U\right] \cap$ $[\Phi \leq M]$. If $x(t) \notin E_{p}$, let $\tau(t)$ be defined by Claim 6.3. Clearly $T \leq \tau(t)<t$ and $x(\tau(t)) \in E_{p}$, which implies that

$$
|x(\tau(t))-p| \leq \rho .
$$

Hence,

$$
\begin{aligned}
h(\tau(t)) & =\langle x(\tau(t))-p, \dot{x}(\tau(t))\rangle+(\gamma / 2)|x(\tau(t))-p|^{2} \\
& \leq \rho\|\dot{x}\|_{\infty}+(\gamma / 2) \rho^{2} .
\end{aligned}
$$

In view of (20) and Claim 6.3, we deduce that

$$
h(t) \leq \rho\|\dot{x}\|_{\infty}+(\gamma / 2) \rho^{2}+\int_{T}^{+\infty}|\dot{x}(u)|^{2} d u .
$$

This boundedness property of $h$ clearly proves that

$$
x \in L^{\infty}([0,+\infty) ; H) .
$$

\section{References}

1. Alvarez, F., On the Minimizing Property of a Second-Order Dissipative System in Hilbert Space, SIAM Journal on Control and Optimization, Vol. 38, pp. 1102-1119, 2000.

2. Aтtouch, H., Goudou, X., and Redont, P., The Heavy Ball with Friction Method, I: The Continuous Dynamical System, Communications in Contemporary Mathematics, Vol. 2, pp. 1-34, 2000.

3. Аtтouch, H., and Czarnecki, M. O., Asymptotic Control and Stabilization of Nonlinear Oscillators with Nonisolated Equilibria, Journal of Differential Equations, Vol. 179, pp. 278-310, 2002.

4. САвот, A., Motion with Friction of a Heavy Particle on a Manifold: Applications to Optimization, Mathematical Modelling and Numerical Analysis, Vol. 36, pp. 505-516, 2002.

5. Hale, J. K., Asymptotic Behavior of Dissipative Systems, Mathematical Surveys and Monographs, American Mathematical Society, Providence, Rhode Island, Vol. 25, 1988.

6. Haraux, A., Systèmes Dynamiques Dissipatifs et Applications, Masson, Paris, France, 1991.

7. BRÉzis, H., Asymptotic Behavior of Some Evolution Systems, Nonlinear Evolution Equations, Academic Press, New York, NY, 1978. 
8. BRuck, R. E., Asymptotic Convergence of Nonlinear Contraction Semigroups in Hilbert Space, Journal of Functional Analysis, Vol. 18, pp. 15-26, 1975.

9. Opial, Z., Weak Convergence of the Sequence of Successive Approximations for Nonexpansive Mappings, Bulletin of the American Mathematical Society, Vol. 73, pp. 591-597, 1967.

10. Савот, A., and Czarnecki, M. O., Asymptotic Control of Pairs of Oscillators Coupled by a Repulsion, with Nonisolated Equilibria, SIAM Journal on Control and Optimization, Vol. 41, pp. 1254-1280, 2002.

11. Aтtouch, H., and Cominetti, R., A Dynamical Approach to Convex Minimization Coupling Approximation with the Steepest Descent Method, Journal of Differential Equations, Vol. 128, pp. 519-540, 1996.

12. Tikhonov, A. N., and Arsenine, V., Méthodes de Résolution de Problèmes Mal Posés, MIR, Moscow, Russia, 1976.

13. Arnold, V., Equations Différentielles Ordinaires, Moscow Editions, Moscow, Russia, 1974.

14. Hirsch, W., and Smale, S., Differential Equations, Dynamical Systems, and Linear Algebra, Academic Press, New York, NY, 1974.

15. Lasalle, J. P., and Lefschetz, S., Stability by Lyapunov Direct Method with Applications, Academic Press, New York, NY, 1961.

16. Reinhardt, H., Equations Différentielles: Fondements et Applications, 2nd Edition, Dunod, Paris, France, 1989. 